\title{
High Rate of Non-Eligibility: Methodological Factors Impacting on Recruitment for a Multicentre, Double- Blind Study of Paediatric Patients with Major Depressive Disorder
}

Authors

Affiliations

\author{
L. Bliznak¹, R. Berg ${ }^{1}$, A. Häge' ${ }^{1}$, R. W. Dittmann ${ }^{2}$
}

${ }^{1}$ Department of Child and Adolescent Psychiatry and Psychotherapy, Research Unit for Paediatric Psychopharmacology, Central Institute of Mental Health, Mannheim, Germany

Eli Lilly Endowed Chair of Paediatric Psychopharmacology, Central Institute of Mental Health, Mannheim, Germany

\author{
Key words \\ - recruitment \\ - eligibility \\ - major depression \\ - paediatric patients
}

$\begin{array}{ll}\text { received } & 06.09 .2011 \\ \text { revised } & 22.03 .2012 \\ \text { accepted } & 03.05 .2012\end{array}$

Bibliography

Dol http://dx.doi.org/

10.1055/s-0032-1314806

Published online ahead of print:

14 June 2012

Pharmacopsychiatry 2013;

46: $23-28$

(c) Georg Thieme Verlag KG Stuttgart · New York

ISSN 0176-3679

Correspondence

\section{Dr. L. Bliznak}

Department of Child and

Adolescent Psychiatry and

Psychotherapy

Research Unit for Paediatric

Psychopharmacology

Central Institute of Mental Health

15

Postbox 122120

68072 Mannheim

Germany

Tel.: +49/(0)621/17034541

Fax: $+49 /(0) 621 / 17034545$

Lucia.Bliznak@zi-mannheim.de

\section{Abstract}

$\nabla$

Introduction: This report describes difficulties encountered when attempting to recruit children and adolescents with major depression for a recent international double-blind, placebocontrolled trial (www.clinicaltrials.gov Nr. NCT 00849901).

Methods: Over a 14-month period, children and adolescents with depressive symptoms were pre-screened for their eligibility for inclusion.

Results: 85 patients (age 7-17 years) were considered. Of these, only one was enrolled. The main reasons for non-eligibility were: failure to meet the baseline severity criterion on the primary outcome scale (Clinical Global Impression

\section{Introduction}

$\nabla$

The importance of clinical trials in child and adolescent psychiatry [1] has increased substantially as a result of the introduction of Regulation 1901/2006 by the European Parliament in 2006, and earlier similar legislation in the United States of America [2]. This legisation has established a system of requirements, rewards, incentives, and horizontal measures to ensure that medicinal products for children and adolescents are adequately researched, developed, and authorised.

The legal framework for conducting clinical trials in the European Union, including those involving children/minors, is set out in the Clinical Trials Directive (CTD) 2001/20/EC. Adherence to the CTD is mandatory for the investigators of all such studies in European paediatric populations [1,3]. Before being authorised for use in adults, a medicinal product must undergo extensive pharmaceutical consistency and stability testing, toxicological tests, and clinical trials to ensure that it is of a high quality, safe, and effective. Until recently, few such studies were performed
- Severity; $32.1 \%$ of the patients); requirement for immediate hospitalisation (15.4\%); or the presence of an exclusionary comorbid psychiatric disorder (19.1\%).

Discussion: The recruitment of paediatric patients with major depression was primarily limited by various inclusion and exclusion criteria. Slow recruitment of small patient samples may impact strongly on the representativeness and generalisability of research findings, and thus on analyses in evidence-based medicine and on the development and recommendations of treatment guidelines. This may impact in turn on the feasibility of the clinical development and registration process of new compounds in paediatric psychopharmacology and beyond.

specifically for children and adolescents. Thus, in contrast to the situation in adults, more than $50 \%$ of all medicines used to treat children and adolescents in Europe have not been authorised for use in paediatric populations [4].

Randomised placebo-controlled or 3-arm clinical trials are now the accepted standard for efficacy studies conducted in order to obtain regulatory approval and market registration. A number of limiting factors may be experienced when conducting such studies, despite careful clinical development of a new compound, thorough planning of study design and protocol, and comprehensive consideration of all aspects of feasibility and implementation.

After selecting a group of potential probands, a second round of screening is required to identify patients who fulfill the inclusion and exclusion criteria and who are able and willing to participate in the trial. In paediatrics as well as child and adolescent psychiatry, the decision to participate in any given clinical study involves both the patient and the parents. 
Barriers to participation may be encountered at each stage of the study recruitment process. Firstly, several aspects of the study design may be considered unacceptable by potential probands or their parents, for example, the demanding and time-consuming nature of the assessments and questionnaires, the randomisation process, or the data handling procedures. The screening procedure per se may also represent a barrier to participation, as this may be viewed by potential participants as a type of intervention in itself [5]. Furthermore, depending on the legal context, informed consent or assent may be required from up to three individuals (the child and both parents) prior to participation in clinical trials in paediatric populations, which often represents an additional organisational hurdle [6]. Relatively few studies have systematically investigated recruitment and related problems in adult as well as child and adolescent psychiatry populations.

Several authors have investigated study-recruitment in adult psychiatric patients. Haberfellner screened 216 depressed adult patients from a private psychiatry practice for their eligibility for inclusion in a randomised double-blind study, and reported a recruitment rate of $0 \%$ due to the failure of patients to meet the eligibility criteria. The author concluded that the inclusion and exclusion criteria that are typically applied in such studies render psychopharmacological studies in office-based practices virtually unfeasible [7]. However, superior recruitment rates have been reported in other psychiatric poplulations. Partonen et al. [8] investigated the recruitment of depressed patients from outpatient psychiatric services, private offices, and health-care centres for a randomised double-blind efficacy trial, and reported a recruitment rate of $34 \%$. They concluded that adult patients with previous depressive episodes or comorbid disorders were less likely to be eligible for inclusion. Greil et al. [9] reported that only $6 \%$ of all screened patients $(N>6000)$ with an affective disorder were enrolled in a long-term prophylactic trial, and discuss the various reasons for non-participation. Bowen et al. [10] reported that nearly $60 \%$ of all patients screened for inclusion in a clinical trial of antipsychotic treatment for acute schizophrenia were ineligible. Of the eligible patients, two thirds refused to provide informed consent, and thus only one in 7 of all patients screened could be included. Meyer zur Capellen et al. [11] retrospectively screened the medical case notes of adult schizophrenia patients from a single large psychiatric hospital to determine whether these patients would have been eligible for inclusion in various clinical trials. The authors reported a potential recruitment rate of $<5 \%$.

With respect to child and adolescent psychiatry, during a 5-year recruitment period for a bulimia nervosa treatment study in adolescents, Hewell at al. [12] reported a randomisation rate of $45 \%$. However, Emslie et al. [13] concluded that children and adolescents can be a difficult population to recruit and retain in clinical studies. For example, they indicated that patients displaying suicidal behaviour or comorbid disorders such as bipolar disorder, substance use disorders, attention deficit/hyperactivity disorder (ADHD), and personality disorders are typically excluded from randomised, controlled trials of treatments for major depressive disorder. In a study of patients from a large academic hospital, Glickman et al. [14] reported that paediatric patients were less likely than adult patients to be enrolled in acute care clinical research studies, and that enrollment rates for paediatric patients were around $40 \%$.

Nonetheless, it should be noted that several successful controlled clinical trials have been performed within the field of child and adolescent psychopharmacology over the last decade. These studies have led in several cases to registration and market authorisation of the investigated compound by the respective regulatory bodies (Food and Drug Administration, European Medicines Agency), e.g., aripiprazole, risperidone, and olanzapine for schizophrenia and bipolar disorder [15-18], atomoxetine for ADHD [19-21], fluoxetine for major depression [22,23], and risperidone for disruptive behaviour in children with below average intelligence $[24,25]$. In general, however, these publications have provided no detailed data on recruitment rates in relation to pre-screening and screening in the respective trials. Beyond recruitment, other methodological issues must be considered in randomised controlled trials in child and adolescent psychiatry populations, for example, higher drop out rates and placebo response rates.

Similar findings to those reported in child and adolescent psychiatry have been reported from studies investigating the recruitment of paediatric patients with somatic disorders. A meta-analysis of 8 acute otitis media trials, for example, reported an estimated recruitment rate across studies of less than $44 \%$ [26]. RECRUIT [27] (processes in recruitment to randomised controlled trials of medicines for children) included 4 trials. In the first study (MASCOT: Management of Asthma in School-aged Children on Therapy), 772 patients were assessed for eligibility. Of these, 54 refused to participate, and 567 patients were excluded. Thus only $19 \%$ of all potential subjects were included. In the second study (MENDS: The use of Melatonin in children with Neurodevelopmental Disorders and impaired Sleep), 241 patients registered for the trial, but 170 refused to participate in the screening process. Thus only $29 \%$ of all potential subjects were included. In the third study (POP: Prevention and treatment of steroid-induced OsteoPenia in children and adolescents with rheumatic diseases), 132 of the 318 patients who had been screened were recruited (42\%). In the fourth study (TIPIT: Thyroxine In Preterm Infants), the parents of 210 children were approached concerning trial participation, and 153 babies were recruited (73\%).

The present study investigated the recruitment of children and adolescents with depressive symptoms for a multicentre randomised study at a German university-based clinical study site. Our aims were to: (i) evaluate the pre-screening-to-recruitment process; (ii) systematically analyse reasons for non-recruitment; and (iii) determine whether severity of illness at pre-screening was a potential moderating factor for successful recruitment.

\section{Patients and Methods \\ $\nabla$}

Over a 14-month period (09/2009-10/2010), children and adolescents with depressive symptoms were pre-screened for their eligibility for participation in an international, multicentre, 3-arm (2 active, 1 placebo), controlled, double-blind efficacy and safety study of paediatric patients with moderate to severe major depressive disorder (www.clinicaltrials.gov Nr. NCT00849901). Protocol-related and other reasons for (non-) eligibility were systematically documented and analysed ( $\bullet$ Table $\mathbf{1}$ ).

Potential study subjects were identified from among patients referred to the child and adolescent psychiatry outpatient department of the Central Institute of Mental Health (CIMH) in Mannheim, or paediatric and child \& adolescent psychiatry practices in the Mannheim area. 
Table 1 Inclusion/exclusion criteria for the randomised trial which included children and adolescents aged 7-17 years who met the criteria for major depressive disorder (as defined in the study protocol).

\begin{tabular}{|c|}
\hline IVI \\
\hline $\begin{array}{l}\text { - outpatient diagnosed with major depressive disorder, as defined by } \\
\text { the Diagnostic and Statistical Manual of Mental Disorders - DSM-IV-TR } \\
\text { ( } 4^{\text {th }} \text { edition, Text Revision) - DSM-IV-TR and supported by the MINI- } \\
\text { International Neuropsychiatric Interview for Children and Adolescents } \\
\text { (MINI-KID) }\end{array}$ \\
\hline - age $7-17$ years \\
\hline $\begin{array}{l}\text { - diagnosis of moderate or severe major depressive disorder as deter- } \\
\text { mined by }\end{array}$ \\
\hline $\begin{array}{l}\text { - CDRS-R: a total score of greater than or equal to } 40 \text { at screening and } \\
\text { randomisation, and a }\end{array}$ \\
\hline $\begin{array}{l}\text { - CGI-Severity rating of greater than or equal to } 4 \text { at screening and } \\
\text { randomisation }\end{array}$ \\
\hline - female patients: negative pregnancy test at screening \\
\hline Most important exclusion criteria \\
\hline $\begin{array}{l}\text { - treatment within the last } 30 \text { days with a drug that has not received } \\
\text { regulatory approval for any indication at the time of study entry }\end{array}$ \\
\hline $\begin{array}{l}\text { - a current or previous diagnosis of bipolar disorder, psychotic depression, } \\
\text { schizophrenia or other psychotic disorder, anorexia nervosa, bulimia, } \\
\text { obsessive compulsive disorder, or pervasive development disorder, as } \\
\text { judged by the investigator }\end{array}$ \\
\hline $\begin{array}{l}\text { - a history of DSM-IV-TR-defined substance abuse or dependence within } \\
\text { the preceding year, excluding caffeine and nicotine }\end{array}$ \\
\hline $\begin{array}{l}\text { - a current primary DSM-IV-TR Axis I disorder other than major depressive } \\
\text { disorder or a current secondary DSM-IV-TR Axis I disorder that requires } \\
\text { any pharmacological treatment }\end{array}$ \\
\hline - one or more first-degree relatives with a diagnosis of bipolar I disorder \\
\hline $\begin{array}{l}\text { - a significant suicide attempt in the year preceding screening or a cur- } \\
\text { rent risk of suicide, in the opinion of the investigator }\end{array}$ \\
\hline $\begin{array}{l}\text { - a positive urine drug screen for any substances of abuse or excluded } \\
\text { medication }\end{array}$ \\
\hline $\begin{array}{l}\text { - current use of any excluded medication (e.g., stimulants or other } \\
\text { antidepressants) }\end{array}$ \\
\hline
\end{tabular}

Table 2 Inclusion/exclusion criteria as the primary reason for not entering the study.

\begin{tabular}{|c|c|c|}
\hline Item & $\begin{array}{l}\text { Non-eligible cases } \\
\mathbf{N}(\text { total } \mathbf{N}=84)\end{array}$ & $\%$ (of cases) \\
\hline $\begin{array}{l}\text { does not meet the baseline } \\
\text { severity criterion (according to } \\
\text { the Clinical Global Impression - } \\
\text { CGl-severity scale) }\end{array}$ & 27 & 32.1 \\
\hline $\begin{array}{l}\text { presence of a comorbid psychi- } \\
\text { atric disorder (e. g., anorexia } \\
\text { nervosa, bulimia, obsessive com- } \\
\text { pulsive disorder) }\end{array}$ & 16 & 19.1 \\
\hline $\begin{array}{l}\text { requires inpatient treatment; } \\
\text { patient hospitalised }\end{array}$ & 13 & 15.4 \\
\hline $\begin{array}{l}\text { history of a significant suicide } \\
\text { attempt in the year preceding } \\
\text { screening or at current risk of } \\
\text { suicide, in the opinion of the } \\
\text { investigator }\end{array}$ & 6 & 7.1 \\
\hline $\begin{array}{l}\text { successful treatment with an- } \\
\text { other antidepressant at the time } \\
\text { of screening }\end{array}$ & 3 & 3.6 \\
\hline $\begin{array}{l}\text { positive urine drug screen for any } \\
\text { substances of abuse or excluded } \\
\text { medication }\end{array}$ & 2 & 2.4 \\
\hline Total & 67 & 79.8 \\
\hline
\end{tabular}

A variety of recruitment strategies was applied. These included informing local office-based paediatricians and child psychiatrists of the study both in writing and during face-to-face educational meetings, placing announcements in local newspapers, and offering information to the public via the CIMH website. Information was provided concerning essential study content and the contact details of study personnel. Physicians and the parents of potential participants were asked to contact the responsible members of the research team by telephone, e-mail, or via the child psychiatry outpatient clinic in order to obtain further information or to arrange an appointment. All study personnel attended the study start-up meeting and attended intensive training in all aspects of the study protocol. These sessions were arranged and funded by the trial sponsor. In most cases, information about the study design and requirements was provided to parents and patients by a fully trained and licensed child and adolescent psychiatrist.

The prescreening process was performed with each family by telephone or in person. This required a period of approximately $30 \mathrm{~min} /$ per family. During this interview, the eligibility of the subject to participate in the trial was assessed on the basis of the main inclusion and exclusion criteria, and all issues pertaining to study participation and the concerns of the parents and the patient were discussed. In a few cases, screening involved the referring physician only, for example when the physician reported a clear exclusion criterion and thus no contact with the family was indicated.

For each potential participant, all reasons for non-participation, including non-eligibility factors, were documented and reported as pre-screening logs to the trial sponsor and to the responsible contract research organisation. For further analyses, only the single main reason for non-eligibility or non-participation per patient was considered. Findings were grouped as "inclusion/ exclusion criteria as reasons for not entering the study" or as "general issues concerning participation in a medication trial as reasons for not entering the study". Reasons for non-participation expressed by the parents and the patients were considered and documented separately. These data were analysed to determine clinical indicators of severity.

Descriptive statistical analyses of the data were performed.

\section{Results \\ $\nabla$}

In the given 14-month period, a total of $\mathrm{N}=85$ paediatric patients were pre-screened for potential recruitment into the trial. These included $\mathrm{N}=51(60.0 \%)$ female and $\mathrm{N}=34(40.0 \%)$ male patients, aged 7.0-17.1 years (mean 13.4 years). In total, $\mathrm{N}=84$ patients could not be enrolled, and one 14-year old female patient was included into the trial. This patient was the $60 \mathrm{~s}$ patient to be screened by our group for this trial.

\section{Non-participation due to failure to meet the study inclusion or exclusion criteria}

Of the 84 non-included subjects, $\mathrm{N}=67$ (79.8\%) either failed to fulfill the trial inclusion criteria or met one or more of the trial exclusion criteria (see $\odot$ Table 2). Of these, $\mathrm{N}=27$ (32.1\%) did not meet the diagnostic severity criterion (i.e., were assessed as not being at least "moderately depressed" according to the Clinical Global Impression - severity scale) [28]. Fulfilled exclusion criteria included the presence of an exclusionary comorbid psychiatric disorder in $\mathrm{N}=16$ (19.1\%) subjects, [e.g., anorexia nervosa in 
Table 3 General issues with participation in a medication trial as the primary reason for not entering the study.

\begin{tabular}{|c|c|c|}
\hline Items & $\begin{array}{l}\text { Non-eligible cases } \\
\mathrm{N}(\text { total } \mathrm{N}=84 \text { ) }\end{array}$ & $\%$ (of cases) \\
\hline $\begin{array}{l}\text { refusal to provide informed } \\
\text { consent }\end{array}$ & 5 & 5.9 \\
\hline - parent & 4 & \\
\hline - patient & 1 & \\
\hline $\begin{array}{l}\text { refusal of any medication to treat } \\
\text { depression }\end{array}$ & 4 & 4.8 \\
\hline - parent & 4 & \\
\hline - patient & 0 & \\
\hline refusal of placebo & 3 & 3.6 \\
\hline - parent & 2 & \\
\hline - patient & 1 & \\
\hline $\begin{array}{l}\text { lack of compliance with study } \\
\text { procedure requirements antici- } \\
\text { pated by investigator }\end{array}$ & 3 & 3.6 \\
\hline $\begin{array}{l}\text { distance of }>200 \mathrm{~km} \text { between } \\
\text { home and study site; feasibility }\end{array}$ & 2 & 2.4 \\
\hline Total & 17 & 20.2 \\
\hline
\end{tabular}

$\mathrm{N}=7$ (8.3\%)], or a positive urine drug screen result in $\mathrm{N}=2(2.9 \%)$. A total of $\mathrm{N}=6$ subjects were excluded due a current risk of suicide or a history of a "significant suicide attempt" within the year preceding the screening procedure.

In the study protocol, a "significant suicide attempt" was defined on the basis of the Columbia Suicide Severity Rating scale [29] as: "a potentially self-injurious act committed with at least some wish to die as a result of the act. Behaviour was in part thought of as method to kill oneself." On this basis, $\mathrm{N}=6$ patients $(8.7 \%)$ were considered to have made a significant suicide attempt in the preceding year or to be at current risk of suicide.

$\mathrm{N}=7$ subjects $(10.1 \%)$ required immediate hospitalisation due to severe depression according to the clinical judgment of the attending physician. $\mathrm{N}=3$ subjects $(4.4 \%$ ) were excluded due to the fact that they were already being successfully treated with another antidepressant at the time of screening.

\section{Non-participation due to general issues}

A proportion of potential subjects did not participate due to concerns about general issues (see $\odot$ Table 3 ). $\mathrm{N}=5$ subjects (5.9\%) refused to provide informed consent. Reasons for refusal included disapproval of scientific studies in general, excessive distance between the subject's home and the study site, or illness of a parent. In 4 of these 5 families, the parents refused to provide informed consent. In the fifth case, the 17-year old female subject refused to participate in the trial. In $\mathrm{N}=4$ cases ( $4.7 \%$ ), the parents refused to agree to any pharmaceutical treatment for depression. In $\mathrm{N}=3$ cases $(4.4 \%)$, the families refused to take the 1 in 3 risk of randomisation to placebo. In 2 of these cases, this was the decision of the parents. In $\mathrm{N}=3$ cases (3.6\%), the patient could not be included due to the expectation of the investigator that the family would not comply with the study procedures. In $\mathrm{N}=2$ cases, participation was not feasible due to the excessive distance $(200 \mathrm{~km})$ between the subjects's home and the study site.

\section{Discussion \\ 7}

The present paper reports systematically collected pre-screening data on a cohort of children and adolescents with depressive symptoms who were ineligible for participation in a 3-arm placebo-controlled registration trial of antidepressant therapy. In contrast to the bulimia nervosa study by Hewell et al. [12], which reported an enrollment rate of $45 \%$ among patients in the United States of America, our enrollment rate was only $1.2 \%$. This may be attributable to differences in the investigated disorder, the exclusion/inclusion criteria applied, or the investigated age group (12-19 years in the study by Hewell et al. compared to 7-17 in the present study). Alternatively, this discrepancy may have been associated with differences in the health-care systems or the routine management of paediatric patients in the respective countries.

Despite the routine application of a variety of recruitment measures and the fact that our child and adolescent psychiatry department is one of the largest in Germany, 14 months were required to identify, and establish contact with, 85 paediatric patients with depressive symptomatology who were potentially eligible for the multicentre trial. Of these, only one patient ultimately participated in the study. Across the other 8 German study centres, only three other cases had been enrolled into the trial by the end of the present 14 month observation period. This finding is consistent with the low recruitment rates reported for a variety of psychiatric disorders in the literature, as reviewed in the Introduction.

Nonetheless, recruitment into the trial across all centres was completed successfully. According to www.clinical trials.gov (NCT00849901; last accessed March 9, 2012), the primary completion date for the multicentre trial was March 2011 (i.e., the final date for data collection for the primary outcome measure), and the study completion date was October 2011. Recruitment figures (no. of patients/site) were also low in other sites and countries, according to informal information obtained by the present authors during the conduct of the multicentre trial. According to clinicaltrials.gov, a total of $\mathrm{N}=337$ patients were recruited at 59 sites in 7 countries ( 24 sites in the USA, and a total of 35 sites in France, Germany, Russia, Ukraine, Slovakia, and South Africa) for the multicentre trial over a total recruitment period of 22 months. Thus, on average, recruitment amounted to $<\mathrm{N}=6$ patients/site or approximately $\mathrm{N}=0.26$ patients/month/site. No further details on prescreening or recruitment were available from this source, and no publications from the trial are available at the time of writing.

Variation in recruitment within this international multicentre trial may have been related to differences in the health-care systems and the routine clinical management of patients in child and adolescent psychiatry in the respective countries. In Germany, for example, relatively more inpatient beds are available compared to other European countries or the United Sates of America. Thus, German paediatric patients with moderate or severe depression are more likely to be treated on an inpatient basis, which ruled out participation in this trial.

In more than $75 \%$ of cases, the patients failed to meet all of the eligibility criteria defined in the study protocol. For example, around $30 \%$ of subjects did not display the required overall symptom severity for major depressive disorder at pre-screening/baseline. This subgroup of patients had to be considered "not severely ill". In contrast, another subgroup of patients presented as being "too severely ill", as reflected in a "history of a 
significant suicide attempt or a current risk of suicide" or a "need for immediate hospitalization", as judged from a clinical perspective (cf. $\bullet$ Table 2 ). In addition, the inclusion of paediatric patients with moderate to severe major depression was likely to be incompatible with the exclusion of fully hospitalised patients (see above). The finding that many patients failed to meet the depression severity criterion is consistent with the findings of Haberfellner [7]. In this study of adult patients, the recruitment rate of $0 \%$ was attributed to the fact that psychiatrists in private practice primarily treat patients suffering from mild depression, dysthymia, or sub-threshold depression. A further reason for non-inclusion cited by Haberfellner was that concomitant treatment was contraindicated. In the present investigation, a small group of potential participants was being treated successfully with another antidepressant at the time of screening. Patients receiving successful treatment with another antidepressant would not be considered candidates for inclusion into a trial, since this would represent an unethical change of therapy.

From an antidepressant efficacy trial, Partonen et al. [8] excluded adult patients with previous depressive episodes or comorbid psychiatric disorders as well as a small group of severely ill patients who presented with suicidality. In our analysis, 6 subjects reported having made a significant suicide attempt in the year preceding the pre-screening assessment. This finding is consistent with the observations of Emslie et al. [13], who stressed that paediatric patients with suicidal behaviour and comorbid psychiatric disorders such as bipolar disorder, substance use disorders, ADHD, and personality disorders are typically excluded from randomised, controlled trials. Almost $20 \%$ of the patients included in our pre-screening process were found to have an exclusionary comorbid psychiatric disorder (primarily anorexia nervosa) and thus could not participate in the trial. Psychiatric comorbidity is common among children treated for depression, in particular externalising disorders such as ADHD. Several types of medication - including psychostimulants were contraindicated by the study protocol, even if the patient was stabilized on that treatment. Since this was a registration trial, the sponsor was obliged to follow standard regulatory body (e.g., European Medicines Agency) requirements in the respective study protocol in order to demonstrate compound-specific efficacy vs. placebo for the primary disorder and to exclude certain adverse-event related risks, such as suicidality. However, the exclusion of patients with comorbid psychiatric disorders from a trial of antidepressants renders recruitment difficult.

Other reasons for non-inclusion were related to general issues concerning participation in a medication trial, such as refusal of the paediatric patient or the parents to provide informed consent/assent. On the basis of clinical observation and long-term experience in implementing paediatric psychopharmacology studies, we assume that refusal to provide consent/assent may also adversely affect the recruitment process of future trials, should informed consent documents continue to appear extremely long and complex, in particular for young patients. The provision of comprehensive information on the study drugs, study procedures, insurance, and data protection - which often reflects institutional review board requirements - plays an important role, as such a large volume of information may be perceived as overwhelming by potential participants. A balance between length and content, as well as (age-) appropriate language, are needed.
The present investigation highlighted important issues relating to the feasibility and implementation of clinical trials in the field of paediatric psychopharmacology. Our findings are limited by the fact that these systematic detailed data on (non)-eligibility were collected from a single German study centre, and relate to a single placebo-controlled 3-arm clinical study of paediatric depression. However, given the paucity of literature on this topic, this report may contribute to an increase in the awareness and knowledge of investigators, sponsors, regulatory bodies, and other involved parties.

Despite the application of a wide range of recruitment approaches and careful prescreening, our study centre achieved a very low rate of recruitment. Thus, assuming that the other trial centres experienced similar limitations, the cohort of this clinical trial of patients with major depression may not have been representative of patients treated in routine clinical practice. When the results of this trial become available, their generalisability may therefore be questionable.

\section{Conclusion \\ $\nabla$}

The present findings suggest that particular inclusion and exclusion criteria limit the recruitment of paediatric patients with major depression into registration studies.

Strict methodological and ethical criteria must be applied for the registration of new compounds, and for the design and implementation of the respective trials. However, slow recruitment of small patient samples may impact strongly on the representativeness and generalisability of the findings of such trials, and thus on (meta-)analyses in evidence-based medicine and on the development and ensuing recommendations of treatment guidelines. If study populations represent only a small percentage of the patient populations that have to be treated in routine clinical practice, study results, combined evidence, and thus evidence-based guidelines that are primarily based on those studies, may not always be predictive and adequate for the majority of the respective patient population. Differences between study findings and clinical experience in terms of the effectiveness and tolerability of compounds may be partly related to these conditions and qualifications.

Limited recruitment and its general impact may also have negative consequences for the feasibility of the clinical development and registration process of new compounds in paediatric psychopharmacology and beyond.

Collection of recruitment data from future trials may enable a more detailed analysis of the issues that affect recruitment. This could facilitate the adoption of alternative trial designs and the avoidance of recruiting difficulties. Close collaboration between the various parties involved in such research, e.g., regulatory bodies, pharmaceutical industry, clinical researchers, and patient/parent advocacy groups, on all aspects of trial design, on the collection of high quality data, feasibility, and representativeness, appears to be warranted and is strongly recommended by the present authors.

\section{Conflict of interest \\ $\nabla$}

The authors declare no conflicts of interest. 


\section{Acknowledgements}

$\nabla$

We would like to thank all of the patients and their parents for their kind support, as well as our colleagues Drs. S. Gerstner, H. Griesemann, B. Kentner-Figura, A. Matei, L. Poustka, B. Rothermel, B. Schillinger, C. Schmäl, and L. Weymann.

\section{References}

1 Lehmann B. Regulation (EC) No 1901/2006 on medicinal products for paediatric use \& clinical research in vulnerable populations. Child Adolesc Psychiatry Ment Health 2008; 2: 37

2 Gruttadaro DE, Miller JE. NAM1 Policy Research Institute Task Force Report Children and Psychotropic Medications. (available on NAMI's web site at www.nami.org).www.fda.gov/ohrms/dockets/dailys/04/ aug04/083104/04n-0330-ts00001-02-vol1.pdf

3 Publication European Commission. Discussions in the Council are progressing well and the proposed Regulation on medicinal products for paediatric use will be discussed at the Health Council of 9 December 2005. [ http:// ec.europa.eu/ enterprise/ pharmaceuticals/ paediat rics/medchild_key_en.htm ]

4 Directive 2001/20/EC of the European Parliament and of the Council of 4 April 2001 on the approximation of the laws, regulations and administrative provisions of the Member States relating to the implementation of good clinical practice in the conduct of clinical trials on medicinal products for human use (1) [ http://europa.eu/eurlex/pri/ en/oj/dat/2001/1_121/1_12120010501en00340044.pdf ]

5 PlueckJ, Freund-Braier I, Hautmann C et al. Recruitment in an indicated prevention program for externalizing behavior - parental participation decisions. Child Adolesc Psychiatry Ment Health 2010; 4: 15

6 Walson PD. Patient recruitment: US perspective. Pediatrics. 1999; 104: 619-622

7 Haberfellner EM. Recruitment of depressive patients for a controlled clinical trial in a psychiatric practice. Pharmacopsychiatry 2000; 33: 142-144

8 Partonen T, Sihvo S, Lonnqvist JK. Patients excluded from an antidepressant efficacy trial. J Clin Psychiatry 1996; 57: 572-575

9 Greil W, Ludwig-Mayerhofer W, Steller B et al. The recruitment process for a multicenter study on the long-term prophylactic treatment of affective disorders. J Affect Disord 1993; 28: 257-265

10 Bowen J, Hirsch S. Recruitment rates and factors affecting recruitments for a clinical trial of a putative anti-psychotic agent in the treatment of acute schizophrenia. Hum Psychopharmacol 1992; 7: 337-341

11 Meyer zur Capellen K, Schmauß M, Messer T. Determinanten für den Ein- und Ausschluss von schizophrenen Patienten bei Phase III- und IV-Studien an einer psychiatrisch-psychotherapeutischen Versorgungsklinik. Available at: edoc.ub.uni-muenchen.de/10477/1/ Meyer_zur_Capellen_Kathrin.pdf

12 Hewell $K$, Hoste R, le Grande $D$ et al. Recruitment for an adolescent bulimia nervosa treatment study. Int J Eating Disord 2006; 39: 594-597

13 Emslie GJ, Ryan ND, Wagner KD et al. Major depressive disorder in children and adolescents: clinical trial design and antidepressant efficacy. J Clin Psychiatry 2005; 66: (Suppl 7): 14-20

14 Glickman SW, Anstrom KJ, Lin L et al. Challenges in enrollment of minority, pediatric, and geriatric patients in emergency and acute care clinical research. Ann Emerg Med 2008; 51: 775-780
15 Findling RL, Robb A, Nyilas $M$ et al. A multiple-center, randomized, double-blind, placebo-controlled study of oral aripiprazole for treatment of adolescents with schizophrenia. Am J Psychiatry 2008; 165: $1432-1441$

16 Findling RL, Nyilas $M$, Forbes $R A$ et al. Acute treatment of pediatric bipolar I disorder, manic or mixed episode, with aripiprazole: a randomized, double-blind, placebo-controlled study. J Clin Psychiatry 2009; 70: 1441-1451

17 Kryzhanovskaya L, Schulz SC, McDougle C et al. Olanzapine versus placebo in adolescents with schizophrenia: a 6-week, randomized, double-blind, placebo-controlled trial. J Am Acad Child Adolesc Psychiatry 2009; 48: 60-70

18 Haas M, Unis AS, Armenteros J et al. 6-week, randomized, double-blind, placebo-controlled study of the efficacy and safety of risperidone in adolescents with schizophrenia. J Child Adolesc Psychopharmacol 2009; 19: 611-621

19 Dittmann RW, Schacht A, Helsberg $K$ et al. Atomoxetine versus placebo in children and adolescents with attention-deficit/hyperactivity disorder and comorbid oppositional defiant disorder: a double-blind, randomized, multicenter trial in Germany. J Child Adolesc Psychopharmacol 2011; 21: 97-110

20 Michelson D, Faries D, Wernicke $J$ et al. Atomoxetine ADHD Study Group. Atomoxetine in the treatment of children and adolescents with attention-deficit/hyperactivity disorder: a randomized, placebo-controlled, dose-response study. Pediatrics 2001; 108: E83

21 Michelson D, Buitelaar JK, Danckaerts $M$ et al. Relapse prevention in pediatric patients with ADHD treated with atomoxetine: a randomized, double-blind, placebo-controlled study. J Am Acad Child Adolesc Psychiatry 2004; 43: 896-904

22 Emslie GJ, Heiligenstein JH, Hoog SL et al. Fluoxetine treatment for prevention of relapse of depression in children and adolescents: a double-blind, placebo-controlled study. J Am Acad Child Adolesc Psychiatry 2004; 43: 1397-1405

23 Emslie GJ, Heiligenstein JH, Wagner KD et al. Fluoxetine for acute treatment of depression in children and adolescents: a placebo-controlled, randomized clinical trial. J Am Acad Child Adolescent Psychiatry 2002; 41: 1205-1215

24 LeBlanc JC, Binder CE, Armenteros JL et al. Risperidone reduces aggression in boys with a disruptive behaviour disorder and below average intelligence quotient: analysis of two placebo-controlled randomized trials. Int Clin Psychopharmacol 2005; 20: 275-283

25 Reyes $M$, Buitelaar J, Toren $P$ et al. randomized, double-blind, placebocontrolled study of risperidone maintenance treatment in children and adolescents with disruptive behavior disorders. Am J Psychiatry 2006; 163: 402-410

26 Bain J. Treatment of acute otitis media: are children entered into trials representative? Br J Gen Pract 2001; 51: 132-133

27 Shilling $V$, Williamson $P R$, Hickey $H$ et al. Processes in recruitment to randomized controlled trials of medicine for children (RECRUIT): a qualitative study. Hlth Technol Assess 2011; 15: 15

28 Busner J, Targum SD. The clinical global impressions scale: applying a research tool in clinical practice. Psychiatry (Edgmont) 2007; 4: 28-37

29 Posner K, Brown GK, Stanley B et al. The Columbia-Suicide Severity Rating Scale: initial validity and internal consistency findings from three multisite studies with adolescents and adults. Am J Psychiatry 2011; 168: 1266-1277 